\title{
Measurements of Porous Networks in Low-k Dielectric by Three-dimensional Electron Tomography
}

\author{
Peter Ercius*, Huolin L. Xin**, and David A. Muller* \\ * School of Applied and Engineering Physics, Cornell University, Ithaca, NY, 14853 \\ ** Department of Physics, Cornell University, Ithaca, NY, 14853
}

The size, distribution and connectivity of the pore network in a porous dielectric determine the electrical and mechanical properties of the overall thin film. The dielectric constant $\kappa$ of the material is typically determined by electrical measurements of capacitive devices, but only indirect measurement methods have been applied to determine bulk physical pore parameters [1]. It is challenging to determine the microscopic parameters of the network as pore sizes approach atomic dimensions, because assumptions based on bulk properties for continuous media are not valid at these length scales. Therefore, a direct determination of the three-dimensional microscopic porous structure is needed to test the properties of low- $\kappa$ dielectrics designed for nanometer-scale integrated circuits.

We report on the use of three-dimensional electron tomography (ET) and annular dark field scanning transmission electron microscopy (ADF-STEM) to reconstruct the porous networks inside a low- $\kappa$ dielectric $(\kappa=2.5)$ with expected $1 \mathrm{~nm}$ pore diameters. ET produces a quantitative reconstruction from a series of projection images acquired at $1^{\circ}$ tilt increments from $\pm 70^{\circ}$ assuming image intensities vary monotonically with the projected material thickness at each raster position [2]. ADFSTEM image intensities vary as the atomic number $Z^{1.7}$ of the target material, which allows spatially resolved material differentiation to determine the boundaries between vacuum and the dielectric matrix for a direct, accurate assessment of the pore network [3]. A restriction of ET for this application is the theoretical $1 \mathrm{~nm}$ resolution limit as determined by the maximum tilt angle achieved and the number of projections acquired [4]. This experiment therefore tests the resolution and contrast limits of ET for nanometer scale investigations of nano-structured materials, and provides a new direct measurement of porosity in a low- $\kappa$ dielectric which is compared to independent observations by the ellipsometric porosimetry bulk measurement technique [5].

Figure 1 shows orthogonal cross-sections approximately $75 \mathrm{~nm}$ across through the three-dimensional reconstruction of the low- $\kappa$ dielectric. Differentiation between the pores and dielectric matrix throughout the sampled volume was accomplished by automatic segmentation with thresholding in the Avizo 5.0 software after determination of the average intensity value at the vacuum/dielectric boundary [6]. The pores do not exhibit a preferred geometry and are randomly distributed throughout the dielectric with no discernible order or orientation. Figure 2 shows the distribution of pore diameters which fits a log-normal distribution with an average of $1.2-1.9 \mathrm{~nm}$, and $95 \%$ of all pores are less than $5.9 \mathrm{~nm}$ across. An independent measurement of the bulk porosity statistics by ellipsometric porosimetry produced similar results, but the ET technique also provides the microscopic distribution of the pores in real space. The porous network contains few large scale, highly connected pores within the dielectric, but only limited pore connectivity is observed overall. Pores with diameters below $1 \mathrm{~nm}$ are not reliably reconstructed due to the resolution limit of ET, and therefore these measurements provide an upper bound on the statistics of the pore network. This direct measurement of the pore size, distribution and connectivity at the nanometer scale by ET 
demonstrates a unique measurement technique for accurate predictions of the electrical and mechanical properties of a porous low- $\kappa$ dielectrics and other nanoscale materials [7].

\section{References}

[1] E. Kondoh, et al., Japanese Journal of Applied Physics, 40 (2001) L323.

[2] P.A. Midgley, et al., Ultramicroscopy, 96 (2003) 413.

[3] E. Kirkland, Advanced Computing in Electron Microscopy, Springer, New York, 1998

[4] J. Frank, Electron Tomography: Methods for Three-Dimensional Visualization of Structures in the Cell, Springer, New York, 2006

[5] M.R. Baklanov, et al., Journal of Vacuum Science and Technology B, 18 (2000) 1385.

[6] Mercury Computer Systems, Avizo 5.1

[7] Research funded by the Semiconductor Research Corporation (SRC) with support from the Cornell Center for Materials Research (CCMR).

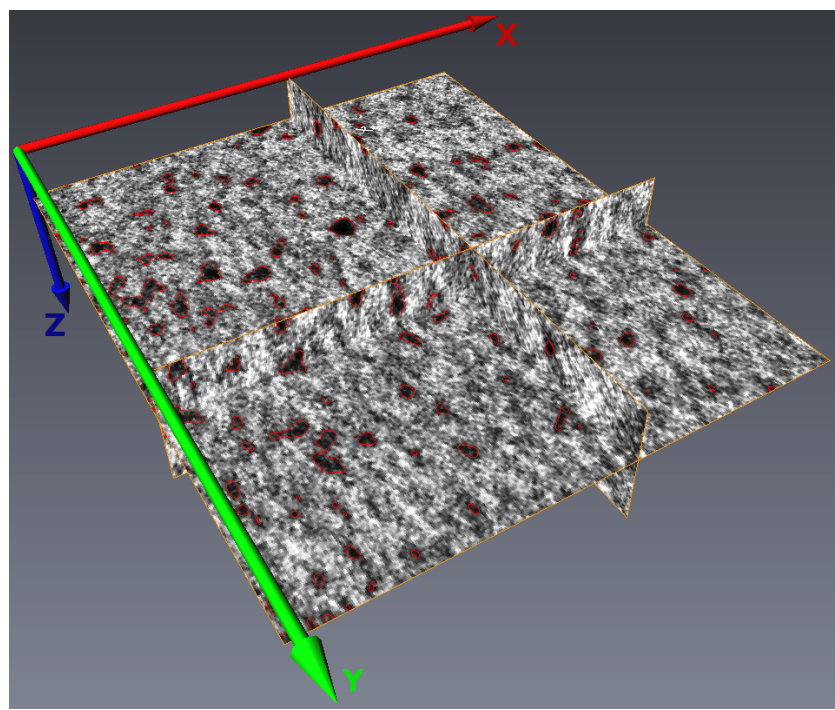

Figure 1: Orthogonal slices approximately $75 \mathrm{~nm}$ across through the three-dimensional reconstruction produced by electron tomography of a porous low- $\kappa$ dielectric showing randomly distributed pores. The network shows little connectivity for the larger scale porosity and the pore diameters follow a log-normal distribution with an average diameter of $1.2-1.9 \mathrm{~nm}$. The reconstruction does not resolve pores $<1 \mathrm{~nm}$ in diameter and therefore only provides information for the larger scale porosity.

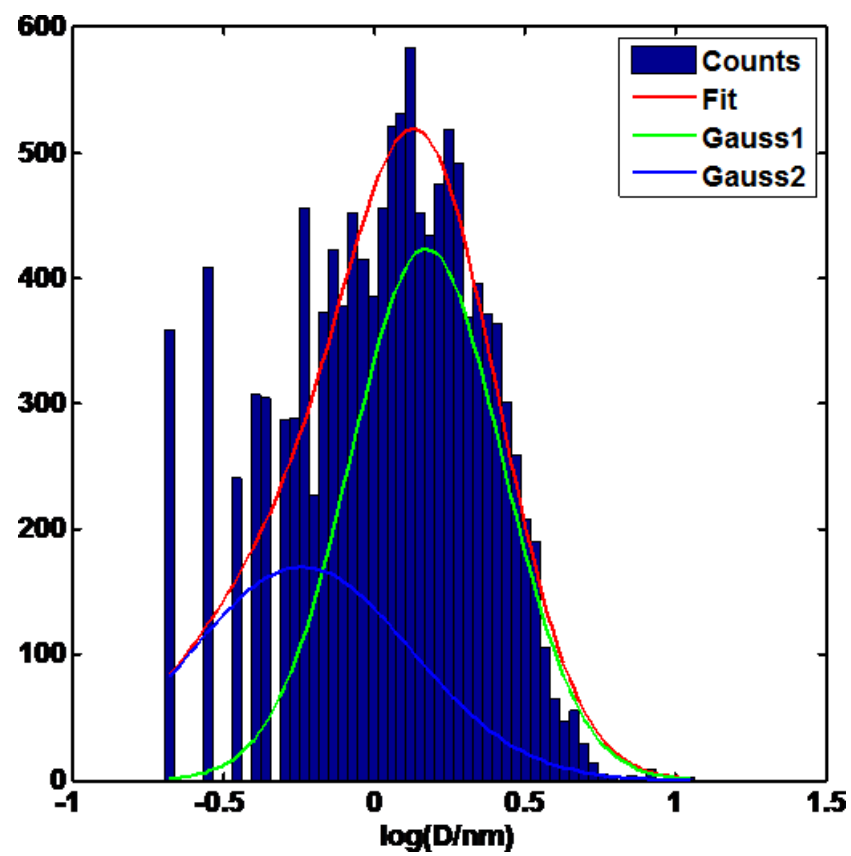

Figure 2. Log-normal distribution of the pore diameters for the porous low- $\kappa$ dielectric as determined by automatic segmentation from an ET reconstruction. The average pore diameter is $1.2-1.9 \mathrm{~nm}$ and $95 \%$ of all pores are less than $5.9 \mathrm{~nm}$ across. The distribution matches independent measurements by ellipsometric porosimetry. 